\title{
Enunciación
}

http://revistas.udistrital.edu.co/ojs/index.php/enunc

\section{Análisis del texto breve extraliterario: elementos para una aproximación conceptual al minitexto}

\author{
Analysis of short non-literary texts: Elements for a conceptual approach to the \\ mini-text
}

\author{
Javier Moreno Caro', Luis Sanabria Rodríguez²
}

Para citar este artículo: Moreno, J., \& Sanabria, L. (2015). Análisis del texto breve extraliterario: elementos para una aproximación conceptual al minitexto. Enunciación, 20(1), pp. 68-77.

Recibido: 25-mayo-2015 / Aprobado: 30-junio-2015

\section{Resumen}

Se propone una aproximación teórica y didáctica al minitexto: categoría conceptual para el análisis y el tratamiento pedagógico de textos breves no literarios. La propuesta se fundamenta en cuatro paradigmas teóricos: uno correspondiente al enfoque bajtiniano del enunciado y los géneros discursivos; otro circunscrito por la lingüística textual y los estudios del discurso; un tercer enfoque relacionado con la teoría y la crítica de la minificción; y un cuarto paradigma que sugiere el aprovechamiento de las características de inmersión y entretenimiento que subyacen a los videojuegos como mecanismos para propiciar aprendizajes significativos.

Palabras clave: enunciado, ficción, cuento, videojuego.

\begin{abstract}
A theoretical and didactic approach to the mini-text is proposed: a conceptual category for analysis and pedagogic treatment of non-literary-short-texts. The proposal is based on four theoretical paradigms: one related to the bakhtinian approach of enunciation and discourse genres; other circumscribed by text linguistics and discourse studies; a third approach related to flash fiction theory and criticism; and a fourth paradigm that suggests taking advantage of immersion and entertainment features that underlay videogames as mechanisms to promote meaningful learnings.
\end{abstract}

Keywords: Statement, fiction, short story, video game.

1 Licenciado en Educación Básica con Énfasis en Humanidades: Español e Inglés, magíster en Tecnologías de la Información Aplicadas a la Educación, Universidad Pedagógica Nacional. Docente catedrático en la Facultad de Posgrados de la Universidad La Gran Colombia, y docente titular adscrito a la Secretaría de Educación del Distrito, Bogotá. Correo electrónico: jomoreno@redp.edu.co

2 Doctor en Educación de la Universidad Pedagógica Nacional, líder del grupo de investigación Cognitek. Correo electrónico: lubsan@gmail.com 


\section{INTRODUCCIÓN}

Minitexto no es un término inédito ni novedoso pero sí algo confuso. Rojo (1997) lo cita como uno de tantos apelativos que solían identificar la narrativa breve en su momento. Era entonces una expresión análoga a los nombres dispuestos en una lista no tan breve: arte conciso, brevicuento, caso, cuento breve y cuento corto. Luego de que Rojo (1997) lo adoptara como la categoría para abarcar todo tipo de narración sumamente breve, Colombo y Tomassini (2000) perfilaron una teoría en torno a los términos de minificción, microtexto y minicuento. En el primer caso, se reconocía el énfasis que el término minificción ponía en "la brevedad y el estatuto ficcional sin hacer alusión a una clase de superestructura discursiva determinada" (p. 26). Este término incluía, entonces, cualquier ficción brevísima sin importar el género o el modo discursivo, por ejemplo, científico o periodístico, argumentativo o narrativo.

En los otros dos casos, microtexto designaba el corpus de ficciones brevísimas en general (casos, obras completas y antologías), en tanto minicuento correspondía a un tipo específico de microtextos en dicho corpus. Según las autoras, minicuento era un término reservado para "aludir a la fecunda subárea integrada por aquellos microtextos donde resulta verificable la presencia de un esquema narrativo subyacente" (p. 27). Tomassini (2007, p. 32) emplearía luego el término de minitexto como sinónimo de microtexto, aludiendo, esta vez, exclusivamente al corpus de microficciones en la obra de Macedonio Fernández. En tanto Tomassini utilizaba el término de minitexto como semblanza del de microtexto, varios autores, según Zavala (2005, p. 54), empleaban el término de minitexto para abordar ya no el corpus de minificciones en general, sino, por el contrario, aquel tipo de texto ultracorto cuya naturaleza no era narrativa.

En este orden de ideas, nos encontramos ante dos definiciones opuestas de minitexto: una referida al corpus de la minificción (cualquier texto o grupo de textos literarios brevísimos, incluidos los minicuentos), la otra relacionada con todas aquellas minificciones que carecen de estructura narrativa. Ante esta dualidad conceptual, proponemos una acepción más de minitexto, a partir de la cual este término gane cierta independencia terminológica respecto a las nociones previamente esbozadas.

El minitexto, como se verá más adelante, abarca aquellos textos breves que pertenecen a los géneros discursivos primarios y a los géneros discursivos secundarios — según la clasificación propuesta por Bajtín (2005)—, haciendo la salvedad de que, dentro de este último grupo, el minitexto se refiere exclusivamente a los géneros extraliterarios. El minitexto, en tanto literatura no ficcional, consiste entonces en enunciados de realidad, ilocuciones serias con un estatuto claramente pragmático; en otras palabras, carece, casi por completo, de lo que Jakobson (1975) denominaría función poética del lenguaje, esto es, "la insistencia en el texto en su forma verbal, forma vuelta con ello más perceptible y en cierto modo intransitiva" (Genette, 1993, p. 21). Entendido así, el minitexto "atiende al dato concreto y se inscribe en el ámbito de la realidad efectiva, de la experiencia empíricamente verificable" (Garrido, 1996, p. 28).

En este punto cabe aclarar que en oposición a la tesis de Roas (2008), para quien el microrrelato no es un género literario autónomo sino "una variante más de la continua reinvención que caracteriza al género cuento" (p. 76), supondremos que el minitexto sí es un género discursivo extraliterario específico, autónomo e independiente; por ende, aquí no será entendido como una variante de los textos no literarios.

Este documento se articula en torno a tres partes: primero, damos cuenta de un estudio cuasiexperimental sobre la comprensión lectora en los videojuegos de rol, a partir del cual se originó esta discusión terminológica a propósito del texto breve extraliterario. En segundo lugar, exponemos las características del minitexto en tanto enunciado, es decir, como unidad de la comunicación discursiva, para luego reseñar las estructuras del minitexto 
desde el enfoque de la lingüística textual y los estudios del discurso. Finalmente, exploramos la potencialidad didáctica del minitexto a partir de su presencia en las nuevas tecnologías de la información y, particularmente, en los juegos digitales.

\section{METODOLOGÍA}

El estudio en cuestión fue de tipo cuasiexperimental con pretest/postest y grupo control, investigación que constó de cuatro condiciones experimentales que dieron lugar a un diseño factorial $2 \times 2$. Dicha investigación buscó contribuir al estudio de los esquemas cognitivos y la comprensión textual, que facilitara el desarrollo de habilidades lectoras fundamentadas en la activación del conocimiento previo del lector utilizando un ambiente lúdico de aprendizaje que permitiera comparar el efecto de dos tipos de esquemas cognitivos en la comprensión de minitextos históricos, pedagógicos y científicos.

En este sentido, el trabajo de investigación constó de tres componentes interdependientes: (i) la comprensión textual en relación con los COnocimientos previos del lector, (ii) una categoría conceptual para describir enunciados breves extraliterarios, y (iii) la propuesta pedagógica para el desarrollo de un ambiente de aprendizaje fundamentado en los videojuegos de rol. Este documento retoma entonces el segundo componente del estudio, a saber, el minitexto (cf. Moreno, 2012).

La selección de minitextos para desarrollar el estudio mencionado se basó en una revisión de diferentes tipos de discurso que, para Colombo y Tomassini (2000), corresponden a los moldes que la minificción toma prestados en calidad de categoría transgenérica: "la fábula, la parábola, el caso, la leyenda, el mito, el cuadro de costumbres, el retrato, la epístola, el aforismo, la sentencia, el informe científico, el aviso publicitario" (Colombo y Tomassini, 2000, p. 26).

Producto de la investigación sobre comprensión lectora y videojuegos de rol (Moreno, 2010), se propuso una teoría acerca del texto breve extraliterario, basada en tres enfoques epistemológicos. El primero permitió abordar el minitexto como enunciado en el eslabón de la comunicación discursiva (Bajtín, 1997, 2005), enunciado que "es producido necesariamente en un contexto particular y siempre es social" (Todorov, 2013, p. 79). El segundo enfoque se fundamentó en la gramática textual (Van Dijk, 1992) para describir el minitexto en términos de sus niveles lingüísticos y estructurales, así como en función de su rol en el proceso interactivo de la comprensión lectora (Van Dijk y Kintsch, 1983). Finalmente, sus principales características fueron definidas a partir de comparaciones con el minicuento y la minificción (Moreno, 2012).

\section{RESULTADOS Y DISCUSIÓN}

\section{El minitexto como enunciado}

En principio, puede decirse que el minitexto es un enunciado verbal de naturaleza eminentemente pragmática. Este rasgo fundamental se debe, en parte, a que responde y a su vez está orientado hacia la respuesta de otros enunciados en la cadena de la comunicación humana. En este sentido, el minitexto tiene principalmente cuatro características, de acuerdo con la propuesta bajtiniana sobre la enunciación discursiva (Bajtín, 1997). Primero, las fronteras del minitexto se constituyen a partir de los intercambios discursivos. Segundo, su naturaleza conclusiva se evidencia en su cierre semántico. En tercer lugar, implica una relación destinataria, pues sugiere un diálogo manifiesto entre los interlocutores y sus producciones discursivas. Finalmente, en calidad de enunciado, se compone de dos partes, una expresada en su realización verbal y otra que consiste en el sobreentendido.

Más allá de los niveles lingüísticos y estructurales, el enunciado le pertenece a un sujeto discursivo, responde a otros, se circunscribe en líneas de interacción con futuros enunciados y limita con todos ellos precisamente porque le pertenece a alguien. Los límites del enunciado pueden 
identificarse en el intercambio discursivo evidente en los distintos "turnos de habla" que ponen al descubierto los participantes de la comunicación discursiva (Bajtín, 2005, p. 264). La segunda característica del minitexto en tanto enunciado es su grado de conclusividad. Para que un enunciado haga parte de la comunicación discursiva, para que pertenezca a alguien y para que pueda dirigirse a otros sujetos discursivos, debe tener sentido para los interlocutores (p. 265). De este modo, el minitexto puede interactuar con los enunciados que le preceden y con aquellos que le esperan.

En la conclusividad del enunciado hay tres factores que se relacionan entre sí: (i) un cierre semántico o agotamiento del sentido que de manera simultánea hace posible vislumbrar las fronteras del enunciado. (ii) La intencionalidad discursiva del hablante (escritor) que determina la elección del objeto, sus límites, la capacidad de agotar su sentido y la elección de la forma genérica que adquirirá dicho enunciado (Bajtín, 2005, p. 266). (iii) La conclusividad del minitexto, que abarca las formas genéricas de conclusión, agrupadas según la esfera discursiva en que se sitúa el enunciado (científica, pedagógica, histórica, judicial, entre muchas otras). Por ejemplo, un enunciado científico puede terminar en una conclusión que sintetice el tema abordado. Un enunciado pedagógico, por su parte, puede concluir exponiendo una enseñanza o una moraleja.

La tercera característica del minitexto es su condición destinataria: su orientación hacia el hablante (escritor) y hacia otros. El enunciado "cobra vida" sociocultural, es decir, se vuelve "un eslabón en la cadena de la comunicación discursiva" (p. 274) cuando se manifiestan en él dos aspectos. Por una parte, la postura activa del hablante dentro de una u otra esfera de objetos y sentidos. De otra, el momento expresivo que representa la actitud subjetiva y evaluadora del interlocutor hacia el contenido semántico de su enunciado, actitud que a su vez implica una evaluación colectiva manifiesta en la relación de los locutores con lo que ocurre (Todorov, 2013, p. 78).
La cuarta y última característica del minitexto como enunciado corresponde a las partes que lo constituyen. En efecto, el enunciado se compone de dos partes, una realizada verbalmente y otra ligada al sobreentendido (Bajtín, 1997, p. 115). La primera parte corresponde a la estructura superficial del texto, es decir, su estructura morfosintáctica y oracional. La segunda parte, el sobreentendido, remite al contexto extraverbal del minitexto, contexto constituido en un horizonte espacial compartido por los hablantes, un horizonte semántico dado en el conocimiento y la comprensión intersubjetiva de la situación del enunciado, y la valoración que los interlocutores hacen de dicha situación.

\section{Entre estructuras y procesos: análisis textual}

Ahora bien, de acuerdo con Van Dijk (1992), un texto es un conjunto de proposiciones que cumple con principios de conectividad para garantizar la cohesión y la coherencia en el discurso. La unidad básica del texto es la proposición, término utilizado por el autor para referirse a redes de conceptos que corresponden a la "idea" que tiene el lector del significado que subyace en la oración, en relación con "una circunstancia posible; en una frase que se expresa en un determinado contexto" (Van Dijk, 1992, p. 40).

La estructura básica del texto es la proposición, pero un texto está conformado generalmente por un conjunto de proposiciones relacionadas entre sí mediante reglas de causalidad, reglas lógicas, situacionales y referenciales (p. 42). De este modo, las proposiciones contenidas en las oraciones que componen un texto se vinculan en estructuras mayores denominadas macroproposiciones.

El siguiente nivel de un texto corresponde a las macroestructuras, es decir, las relaciones globales o temáticas que se refieren a las transformaciones semánticas de que son objeto las proposiciones de un texto. Dichas transformaciones permiten que las oraciones del texto se vinculen localmente y proporcionan las bases para su integración en unidades mayores. En este sentido, 
las macroproposiciones se consolidan a partir de reglas para omitir información no relevante e integrar otras proposiciones mediante operaciones de generalización y construcción (Van Dijk, 1992, p. 199).

Finalmente, los textos se organizan de acuerdo con superestructuras específicas, es decir, formas genéricas del discurso que definen la ordenación global de este y la relación entre sus partes (Van Di$j k, 2000)$. De este modo, la superestructura orienta la organización de micro y macroproposiciones de acuerdo con categorías convencionalmente compartidas: introducción, cuerpo, conclusión, argumento, moraleja, complicación, etc. (Van Dijk, 1998, p. 52).

\section{En el marco de la minificción}

Luego de definir algunas características del minitexto que en nada lo diferencian de cualquier texto no literario, enunciaremos ahora aquellos rasgos que lo individualizan, le proporcionan autonomía y lo identifican, en cierta medida, con la minificción. Con este propósito reseñaremos las propuestas de tres teóricos de la minificción para, luego, señalar las diferencias y similitudes entre esta y el minitexto.

Violeta Rojo (1997) define de manera específica el minicuento como una narración breve de ficción, en la que personajes y acciones se exponen de manera rigurosa y económica. De acuerdo con la autora, el lenguaje del minicuento es elíptico y condensado, posee carácter proteico, adopta distintas formas genéricas y establece relaciones intertextuales. Por su parte, Colombo y Tomassini (2000) definen la minificción como una clase textual transgenérica que incluye tres características fundamentales: primero, su breve extensión; segundo, una textura cimentada sobre la retórica de la omisión y la condensación; tercero, un tipo particular de interacción que promueve su lectura.

Lauro Zavala (2004), uno de los investigadores más prolíficos de la minificción hispanoamericana, plantea algunos criterios de clasificación de acuerdo con la extensión de los textos: cuentos cortos (1.000 a 2.000 palabras), cuentos muy cortos (200 a 1.000 palabras) y cuentos ultracortos ( 1 a 200 palabras), sus características en razón del vínculo estructural entre la parte y el todo, y los problemas que enfrenta la minificción en relación con la teoría, la lectura, la publicación, el estudio y la escritura (Zavala, 2005).

Zavala (2004) propone, en primer lugar, la estética de cuentos cortos (1.000 a 2.000 palabras), cuentos que han sido reunidos en diversas antologías bajo el nombre de ficción súbita, cuentos microcósmicos o short shorts. En el marco de los cuentos cortos, retoma los planteamientos de Irving Howe y Charles Baxter para señalar ciertas coincidencias temáticas, a saber: (i) un incidente repentino que produce epifanías despojadas de su respectivo contexto; (ii) la condensación de toda una vida encapsulada en una imagen paradigmática; (iii) una imagen instantánea en la que se presenta un monólogo interior o un flujo de memoria; (iv) una estructura alegórica que, dada su belleza, impide su interpretación; y (v) la reacción de un personaje ante un momento de tensión súbita.

En segundo lugar, se refiere a los cuentos muy cortos (200 a 1.000 palabras) como una categoría constituida por los textos usualmente denominados como flash fiction, compilaciones de microhistorias y narraciones instantáneas que rompen la linealidad de la secuencia narrativa mediante recursos elípticos y metafóricos. En el primer caso se omiten fragmentos del relato a través de la intensificación del tiempo, recurriendo a incidentes repentinos o a la condensación de una vida. En el segundo caso, algunos fragmentos del relato son sustituidos por elementos inesperados y disonantes tales como el monólogo interior y la estructura alegórica.

En tercer lugar, los cuentos ultracortos (1 a 200 palabras) están formados por fragmentos narrativos elegidos para distintas selecciones temáticas (Borges y Bioy-Casares, 1976), compilaciones (Valadés, 2001), minicuentos, casos y, entre muchos otros, textículos (Cortázar, 2010). El cuento 
ultracorto se caracteriza por incluir diversas estrategias de intertextualidad (hibridación genérica, alusión, parodia), diversas clases de metaficción (metalepsis, diálogo con el lector, repeticiones lúdicas), diversas clases de ambigüedad semántica (final sorpresivo o enigmático) y diversas formas de humor (intertextual) e ironía inestable (Zavala, 2004, p. 99).

Ahora bien, respecto a las características de la minificción en razón al vínculo entre la parte y el todo, Zavala (2005) se refiere al fragmento como la ruptura de una totalidad en elementos que conservan una autonomía textual, y al detalle como la segmentación provisional de una unidad global, íntegra e indivisible. El fragmento y el detalle son entonces mecanismos de unidad y fragmentación denominados estrategias de serialidad, que pueden ser de carácter anafórico (se retoma un hecho anterior), catafórico (anuncia un hecho por ocurrir), elipsis (suprime un hecho que se da por ocurrido), analepsis (flashback) y prolepsis (flashforward).

Finalmente, la minificción se caracteriza en relación con seis atributos que definen este género del tercer milenio: (a) brevedad (una escritura que no excede el espacio convencional de la cuartiIla o la página impresa); (b) diversidad, dada en la naturaleza híbrida de su estructura interna y en la proliferación de géneros a los que la minificción se aproxima; (c) complicidad, en tanto acto nominativo que acarrea la responsabilidad de fijar un nombre a un género proteico; (d) fractalidad, puesto que la minificción acoge tanto al detalle como al fragmento en calidad de estrategias de serialidad; (e) fugacidad, respecto a la dimensión estética de la minificción a la luz de la crítica y la teoría literaria; y (f) virtualidad, puesto que en muchos casos la minificción implica el uso de un programa interactivo frente al cual el lector no solo interpreta sino que participa a modo de coautor, interviniendo la estructura y el lenguaje del texto mismo (Zavala, 2005).

A propósito de las similitudes entre el minitexto y los discursos estéticos breves correspondientes a la minificción, cabe señalar cinco características del texto breve que nos ocupa: (i) la extensión del minitexto, al igual que la del cuento ultracorto, no excede la página impresa o "el pantallazo" (sin importar cuál sea el dispositivo electrónico: smartphone, tableta, PC o consola de videojuegos); en otras palabras, el minitexto abarca todos aquellos textos breves no literarios cuya composición oscila entre 1 y 200 palabras. (ii) El lenguaje utilizado en los minitextos es claro y cumple funciones pragmáticas específicas, aunque las proposiciones que integran un minitexto también pueden expresarse de forma elíptica y condensada. (iii) El minitexto también alude a la diversidad en tanto rasgo característico, por cuanto abarca innumerables géneros primarios y secundarios; (iv) también recurre a diferentes estrategias de serialidad y, por ende, se caracteriza por su fractalidad y, finalmente, (v) está estrechamente ligado a las nuevas tecnologías en razón de su virtualidad.

\section{De los géneros discursivos}

Ahora bien, una tipología preliminar sobre el minitexto puede enunciarse en los siguientes términos. Los géneros del minitexto corresponden a esferas específicas de la comunicación discursiva. En otras palabras, para cada actividad social existen géneros discursivos que se caracterizan por su contenido, estilo verbal, composición y estructura. Siguiendo a Bajtín (2005), "cada esfera del uso de la lengua elabora sus tipos relativamente estables de enunciados, a los que denominamos géneros discursivos" (p. 248).

Por ejemplo, las interacciones plausibles en una entidad bancaria están mediadas discursivamente en relación con textos específicos como recibos para consignar dinero, formularios para solicitar créditos y formatos para abrir cuentas bancarias. Igualmente, los tipos relativamente estables de enunciados en una esfera judicial pueden corresponder a formatos para realizar declaraciones juramentadas, confesiones, denuncias, etcétera. De acuerdo con Bajtín (2005), una clasificación inicial de los enunciados corresponde a la distinción 
entre géneros primarios (simples) y géneros secundarios (complejos). Los géneros discursivos primarios son los enunciados que corresponden a las relaciones discursivas entre los interlocutores en la vida diaria. Por ejemplo, los diálogos cotidianos, familiares e íntimos son enunciados primarios.

Por su parte, los géneros discursivos secundarios, según Bajtín, "surgen en condiciones de la comunicación cultural más compleja, relativamente más desarrollada y organizada, principalmente escrita" (p. 250). Los géneros secundarios se dividen a su vez en géneros literarios y géneros extraliterarios. Puede decirse que la diferencia fundamental entre unos y otros es la intención manifiesta en dichos enunciados. Los géneros literarios tienen principalmente una función estética, en tanto los extraliterarios tienen una función claramente pragmática.

En esta instancia podemos afirmar que los minitextos bien pueden pertenecer tanto al género primario como al género secundario. Respecto al primer caso, basta imaginar los diálogos cotidianos articulados mediante dispositivos digitales tales como el correo electrónico o las aplicaciones de mensajería instantánea (i. e., WhatsApp o Facebook). En relación con el género secundario, los minitextos corresponden a enunciados complejos que, recurriendo a diferentes estrategias de serialidad, pueden ser transformados y compartidos a través de diversas herramientas virtuales (i. e., servicios de microblogging como Twitter o redes sociales como Facebook), acoplándose así a esferas de comunicación mucho más complejas.

En este orden de ideas, en la actualidad existe un gran número de minitextos enmarcados en lo que Cassany (2008) Ilamaría "nuevas prácticas letradas". Este autor cita a Emilia Ferreiro para resaltar que leer y escribir son construcciones sociales con circunstancias históricas particulares, que dan origen a nuevos sentidos y modos de enunciación discursiva. Ejemplos del anterior planteamiento son los minitextos instructivos e informativos que yacen en máquinas expendedoras, señales de tránsito y vallas publicitarias. Estos suelen abarcar, siguiendo a Cassany, indicaciones sobre transporte, destinos turísticos y edificaciones singulares.

Igualmente, los teléfonos inteligentes y las herramientas virtuales para la comunicación en red han dado lugar a la proliferación de nuevos textos que se caracterizan por su brevedad, elipsis, condensación y virtualidad. Tal es el caso de los minitextos elaborados a partir de la combinación de caracteres no convencionales que expresan emociones: felicidad [:)], tristeza [:(], etcétera. De tal forma, esta categoría incluiría los mensajes de texto que pueden ser enviados y recibidos desde teléfonos móviles; mensajes que transitan de uno a otro correo electrónico y, entre muchos otros, mensajes escritos mediante sistemas de comunicación instantánea y redes sociales.

Al género secundario también pertenecen minitextos como los mensajes publicitarios. Estos minitextos pueden ser impresos o digitales, y suelen apoyarse en otros códigos de expresión como el dibujo, la animación o la fotografía. Existen además minitextos históricos que incluyen la crónica, el reportaje periodístico y la biografía sintética (Borges, 2005); minitextos científicos que abarcan las reseñas bibliográficas, los artículos enciclopédicos y los microensayos (Diaz, 1995) y, entre muchos otros, los minitextos pedagógicos, allí donde han ocupado un importante lugar, a lo largo de la historia, textos breves como la fábula, la parábola, el ejemplo y la instrucción.

No sobra agregar dentro de esta tipología del minitexto, aquellas producciones culturales que han sido abordadas a la luz de la minificción audiovisual. Tal es el caso de la publicidad televisiva, la animación experimental y el avance cinematográfico (Zavala, 2008). En esta misma corriente se ubican los estudios acerca de los créditos cinematográficos, así como de las secuencias de apertura y cierre en los seriados televisivos y las películas (Zavala, 2014). Los seriados animados tampoco quedan exentos de esta breve tipología, aunque estos tiendan a ubicarse más cerca de la minificción audiovisual que del minitexto en particular (Moreno, 2015). 


\section{A MODO DE CONCLUSIÓN: APORTES PARA UNA DIDÁCTICA DEL MINITEXTO EN LA ERA DIGITAL}

Hasta acá hemos delimitado el concepto de minitexto en el marco de algunos enfoques sobre la minificción, sugiriendo esta vez una acepción del término en calidad de texto breve extraliterario. Adicionalmente, sintetizamos las características que permiten definir el minitexto en tanto enunciado, así como las estructuras textuales que lo constituyen a la luz de los estudios del discurso. Luego de reseñar algunas definiciones del minicuento y la minificción para abordar obvias similitudes entre el minitexto y dichos discursos estéticos expusimos una tipología preliminar fundamentada en la propuesta de Bajtín (2005) sobre los géneros discursivos.

Ahora señalaremos dos elementos para una didáctica del minitexto. El primero, orientado al desarrollo de la comprensión y la producción textual, corresponde a la interactividad como imprescindible atributo de cualquier hipermedia, educativo o no. El segundo, por su parte, se refiere a un componente indispensable para cualquier propuesta pedagógica basada en el juego y la lectura, a saber: el ensimismamiento. En el caso de la interactividad, existen hoy plataformas para la administración de cursos educativos (i. e., Blackboard o Moodle) que, mediante herramientas como wikis, foros y chats, favorecen la construcción colectiva de textos breves. Este tipo de ambientes, aún sin involucrar patrones lúdicos en su estructura y funcionamiento, se constituyen en objetos virtuales de aprendizaje que permiten desarrollar procesos de lectoescritura basados en el trabajo en equipo, la sociabilidad, el pensamiento crítico y la autonomía discursiva.

Adicionalmente, la interactividad es un rasgo que caracteriza a cualquier tipo de juego, digital o no, pues este necesita de la participación del jugador para hacerse realidad. Es así como los videojuegos se constituyen en laboratorios de experimentación emocional y social que, siguiendo a Aranda y Sánchez-Navarro (2009), permiten explorar sensaciones, estrechar vínculos sociales y crear redes para el intercambio material y de conocimiento. Según estos autores, los videojuegos también pueden usarse en el campo del aprendizaje por tres razones: en ellos confluyen las necesidades y el estilo de los estudiantes actuales, son motivadores en tanto divertidos y son versátiles y adaptables.

Por eso los videojuegos permiten innovar en nuevas formas de experimentar con el entorno, en la interacción con otros individuos y como herramientas para construir una identidad en libertad, así como para adoptar identidades alternativas. De tal forma, el videojuego y el juego en general se entienden como máquinas de aprendizaje, como textos que se usan, como relatos que se leen y como artefactos que se viven. Por supuesto, no hay que olvidar que este tipo de ambientes fomenta la concentración, el interés por el descubrimiento y el mejoramiento de distintas competencias, características ampliamente explotadas por el aprendizaje basado en juegos (Game-based Learning).

Finalmente, el segundo elemento para el desarrollo de una didáctica del minitexto basada en los videojuegos remite al carácter lúdico de la lectura y, muy especialmente, al grado de inmersión que el lector-jugador Ilega a experimentar al momento de sumergirse en un juego digital. De hecho, cuando los participantes de un juego se involucran en este, llegan a tal grado de ensimismamiento que pierden la noción del tiempo, del espacio e incluso de ellos mismos. Casualmente, cuando una persona lee se generan ciertas condiciones que la llevan a un grado de abstracción del mundo muy similar al experimentado por un jugador. De acuerdo con Graciela Montes (2001), cuando se juega, al igual que cuando se lee, se está en otra parte, se cruza una frontera, se está en un espacio significativo y en un lapso temporal en los que sucede algo intenso y gratuito. En dicho momento, la lectura pasa a ser un placer, un pasatiempo intrascendente $y$ divertido.

Por consiguiente, la lectura y el juego digital están estrechamente ligados: ambos representan 
actividades gratuitas que ocasionan placer en sí mismas, son actividades generadas en dimensiones espaciales y temporales distintas a la cotidianidad, crean orden a partir del caos (Huizinga, 2000), tienen sentido propio y generan en el lector, al igual que en el jugador, una sensación de ensimismamiento. De manera similar, Mendizábal (2004) aborda la eficacia de los videojuegos por cuanto crean un espacio sicológico y mental que llevan a que el jugador se escinda del espacio real. En consecuencia, el videojuego como producto de las nuevas tecnologías permite la desterritorialización, la deslocalización y la deshistorización a través del individualismo, el sedentarismo personal y la creación de microrrelatos.

Tal grado de inmersión propio del juego y la lectura corresponde a una categoría acuñada previamente para describir el placer experimentado por artistas y músicos al aislarse del mundo que los rodea, un movimiento de "vaivén" que no está vinculado a fin alguno (Gadamer, 1991). Este concepto, aplicable por igual para describir el ensimismamiento en la lectura y en el videojuego, define un estado mental al que según Aranda y Sánchez-Navarro (2009), se acerca el jugador gracias a las dinámicas adaptativas e interactivas del videojuego.

Para concluir traemos a colación una minificción de Abraham Dantus, incluida por Edmundo Valadés en El libro de la Imaginación, que sintetiza en algo esta última idea:

\section{Ensimismamiento}

A la hora del cofi-breik bajé a la cafetería de la planta, pedí un café. Estaba cansado de la rutina y de mi trabajo, tenía que descansar.

Mientras sorbía lentamente mi café me puse a observar una pintura marina, bastante mala, que colgaba de la pared.

Pensé en Acapulco, puestas de sol, Puerto Escondido, me iba absorbiendo cada vez más y más.

Salí de este ensimismamiento cuando una ola mojó mis pies.

\section{RECONOCIMIENTOS}

Este artículo se derivó de la investigación "Comprensión de Minitextos a partir de la Activación de Esquemas Cognitivos en un Videojuego de rol" (2010), requisito presentado para obtener el título de magíster en Tecnologías de la Información Aplicadas a la Educación de la Universidad Pedagógica Nacional. Esta investigación se planteó en el marco de las políticas para el desarrollo de proyectos educativos que fortalecieran las capacidades lectoras de los estudiantes e involucraran el uso de las TIC en las instituciones educativas del país. En este sentido se proyectó un estudio como alternativa didáctica de implementación tecnológica, para abordar la comprensión de textos breves no literarios en un videojuego de rol. La realización de este artículo ha contado con el apoyo de la Universidad Pedagógica Nacional y el grupo de investigación Cognitek, entidades que han facilitado el desarrollo de los procesos de formación e investigación en educación posgradual, conduciendo a la realización de la propuesta educativa y la investigación de las que trata este artículo. Así mismo, el trabajo y la colaboración de las directivas, los maestros y los estudiantes de la I. E. D. Colegio Isabel II contribuyeron significativamente para que el estudio en cuestión llegara a buen término.

\section{REFERENCIAS}

Aranda, D., y Sánchez-Navarro, J. (2009). Aprovecha el tiempo y juega. Algunas claves para entender los videojuegos. Barcelona: Editorial UOC.

Bajtín, M. (1997). Hacia una filosofía del acto ético. De los borradores y otros escritos. Barcelona: Anthropos.

Bajtín, M. (2005). Estética de la creación verbal. México: Siglo XXI.

Borges, J. L. (2005). Textos cautivos. Madrid: Alianza.

Borges, J. L., y Bioy-Casares, A. (1976). Cuentos breves y extraordinarios. Buenos Aires: Losada.

Cassany, D. (2008). Prácticas letradas contemporáneas. México: Ríos de Tinta. 
Colombo, S. y Tomassini, G. (2000). Comprensión lectora y producción textual: minificción hispanoamericana. Rosario: Editorial Fundación Ross.

Cortázar, J. (2010). Último round. México: Editorial RM.

Diaz, L. (1995). Atisbos en el crepúsculo. Cúcuta: Fondo Mixto de Promoción de la Cultura y las Artes de Norte de Santander.

Gadamer, H. G. (1991). La actualidad de lo bello. Barcelona: Paidós.

Garrido, A. (1996). El texto narrativo. Madrid: Síntesis, S. A. Genette, G. (1993). Ficción y dicción. Barcelona: Lumen. Huizinga, J. (2000). Homo Ludens. Madrid: Alianza.

Jakobson, R. (1975). Ensayos de lingüística general. Barcelona: Seix Barral.

Mendizábal, I. (2004). Máquinas de pensar: videojuegos, representaciones y simulaciones de poder. Quito: Abya-Yala.

Montes, G. (2001). La frontera indómita: en torno a la construcción y defensa del espacio poético. México: Fonde de Cultura Económica.

Moreno, J. (2010). Comprensión de minitextos a partir de la activación de esquemas cognitivos en un videojuego de rol. (Tesisde Maestría). Bogotá: Universidad Pedagógica Nacional.

Moreno, J. (2012). Juguemos en la clase mientras el profe está: comprensión lectora y videojuegos de rol. Bogotá: Universidad La Gran Colombia.

Moreno, J. (2015). It's a Mad, Mad, Mad, Mad Cartoon: para un análisis transtextual de la minificción audiovisual. En A. Rueda (Ed.), Actas del VIII Congreso Internacional de Minificción (en prensa). Lexington: Iberoamericana-Vervuert.
Roas, D. (2008). El microrrelato y la teoría de los géneros. En I. Andres-Suárez y A. Rivas (Eds.), La era de la brevedad: el microrrelato hispánico (pp. 47-76). Palencia: Menoscuarto ediciones.

Rojo, V. (1997). Breve manual para reconocer minicuentos. México: Universidad Autónoma Metropolitana.

Todorov, T. (2013). Mijaíl Bajtín: el principio dialógico. Bogotá: Instituto Caro y Cuervo.

Tomassini, G. (2007). La microficción en la obra de Macedonio Fernández. El Cuento en Red, 20-35.

Valadés, E. (. (2001). El libro de la imaginación. México, D. F.: Fondo de Cultura Económica.

Van Dijk, T. (1992). La ciencia del texto. Buenos Aires: Paidós.

Van Dijk, T. (1998). Estructuras y funciones del discurso. México: Siglo XXI.

Van Dijk, T. (2000). El discurso como estructura y proceso. Barcelona: Gedisa.

Van Dijk, T., y Kintsch, W. (1983). Strategies of discourse comprehension. New York: Academic Press.

Zavala, L. (2004). Cartografías del cuento y la minificción. Sevilla: Renacimiento.

Zavala, L. (2005). La minificción bajo el microscopio. Bogotá: Universidad Pedagógica Nacional.

Zavala, L. (2008). La minificción audiovisual. En I. Andres-Suárez, y A. Rivas (Eds.), La era de la brevedad (pp. 207-230). Palencia: Menoscuarto.

Zavala, L. (2014). Los créditos cinematográficos como minificción. En H. González (Ed.), La minificción en el siglo XXI: aproximaciones teóricas (pp. 138149). Bogotá: Universidad Nacional de Colombia. 